\title{
Molecular Gas in the Magellanic Clouds
}

\author{
Mónica Rubio \\ Departmento de Astronomía, Universidad de Chile, Casilla 36-D, \\ Santiago, Chile
}

\begin{abstract}
The molecular gas content in the Magellanic Clouds has been studied, with different spatial coverage and resolution, through obervations of $\mathrm{CO}(1-0)$ line emission. In the $\mathrm{LMC}$ and the SMC the molecular gas is dominated by clouds whose properties are different from those of their Galactic counterparts. The relation between the intensity of $\mathrm{CO}$ emission and molecular hydrogen column density, or the conversion factor $X$, is different than that of molecular clouds in our Galaxy and depends on the ambient physical conditions. Studying the molecular gas through observations in the $\mathrm{H}_{2}$ emission line may prove an alternative way to determine the molecular content associated with star forming regions in the Magellanic Clouds. In particular, results obtained towards 30 Doradus in the LMC are presented.
\end{abstract}

\section{Introduction}

The Large and Small Magellanic Cloud (LMC, SMC), the two nearest extragalactic systems of well known distance provide a unique opportunity to study physical processes in an interstellar medium different from that of our Galaxy. They have lower metallicities, higher gas to dust ratios and strong UV radiation field, and hence constitute the best target for a detailed study of the molecular gas content and physical properties, and the study of star formation processes associated with them.

In our Galaxy, extensive observations of the $\mathrm{CO}$ emission have shown that molecular gas $\left(\mathrm{H}_{2}\right)$ rather than atomic gas $(\mathrm{HI})$ is the most active component of the interstellar medium. The molecular gas appears concentrated in massive $\left(10^{6}\right.$ $\mathrm{M}_{\odot}$ ) giant molecular clouds which are the sites of all known regions of massive and luminous stars (Combes 1991). Extragalactic CO observations have shown that molecular clouds within the disk of late type spirals appear to share the same characteristics as those of our Galaxy (Young \& Scoville 1991).

A different situation is seen in irregular galaxies. The observations show a strong defficiency in $\mathrm{CO}$ emission compared to spirals of the same luminosity and star forming activity (Rubio et al. 1991 and references therein). Based on the critical assumption that the conversion factor used to derive molecular hydrogen masses from the observed CO emission is the same as in our Galaxy, these galaxies have been claimed to contain negligible amounts of molecular gas. On the other hand, irregular galaxies contain giant regions of ionized gas (HII regions) and luminous and massive stars (OB associations), whose presence 
suggests episodes of recent star formation. Therefore several questions are raised by the apparent contradiction between a high rate of star formation and the absence of molecular material.

Thus, a major goal of the study of the molecular gas content in the Magellanic Clouds has been to determine the relation between $\mathrm{CO}$ and $\mathrm{H}_{2}$ under conditions significantly different from those found in our Galaxy. Another important aspect to be addressed, since stars form in molecular clouds, is the study of star formation processes in these low metallicities molecular clouds.

\section{Millimeter-wave Studies of the Molecular Gas}

Molecular gas in the Magellanic Clouds has been investigated from observations of the tracer molecule $\mathrm{CO}$ made with different spatial resolutions and coverages. The first complete $\mathrm{CO}(1-0)$ survey of the Magellanic Clouds was made using the Columbia Southern Telescope at Cerro Tololo with an 8'.8 resolution (150 pc linear resolution) and covered an area of $6^{\circ} \times 6^{\circ}$ for the LMC (Cohen et al. 1988 ) and $3^{\circ} \times 2^{\circ}$ for the SMC (Rubio et al. 1991).

The ESO-SEST Key Programme: CO in the Magellanic Clouds (Israel et al. 1993; Rubio 1997) has produced a wealth of CO observations of the LMC and $\mathrm{SMC}$, with an angular resolution of $45^{\prime \prime}$ at $115 \mathrm{GHz}(\sim 10$ pc linear resolution). The SEST telescope fully resolves the giant molecular clouds, allowing the determination of their sizes and critical parameters to obtain virial masses, which can be used in turn to calibrate the conversion factor between molecular hydrogen $\mathrm{H}_{2}$ and $\mathrm{CO}$ emission.

A new complete $\mathrm{CO}(1-0)$ survey of the Large Magellanic Clouds has been recently undertaken with the $4 \mathrm{~m}$ NANTEN radiotelescope at Las Campanas Observatory (Fukui et al. 1999). This survey, made with angular resolution of $2.6,(\sim 30$ pc linear resolution $)$ covers the same $6^{\circ} \times 6^{\circ}$ area previously surveyed at 8 '.8 by the Columbia survey.

\section{Results}

The Columbia surveys showed the overall distribution of the molecular gas in both clouds. The $\mathrm{CO}$ emission appeared associated with $\mathrm{HII}$ and radio continuum regions and was detected towards the highest column densities of $\mathrm{HI}$ : $\mathrm{N}(\mathrm{HI})>10^{21} \mathrm{~cm}^{-2}$ in the LMC and $\mathrm{N}(\mathrm{HI})>10^{22} \mathrm{~cm}^{-2}$ in the SMC. In the LMC, about 40 complexes were identified with typical sizes of $\sim 200 \mathrm{pc}$ and $\mathrm{CO}$ luminosities of $\sim 1 \times 10^{5} \mathrm{~K} \mathrm{~km} \mathrm{~s}^{-1} \mathrm{pc}^{-2}$. The CO complexes appeared to be underluminous in $\mathrm{CO}$ as compared to galactic GMC's with similar velocity line widths. Based on the observed $L_{C O}-\Delta v$ relationship, Cohen et al. (1988) and Rubio et al. (1991) suggested that the conversion factor to derive $\mathrm{H}_{2}$ column densities from CO luminosities was a factor of 6 and 20 larger than the Galactic factor in the LMC and SMC, respectively. Using the derived factors, the total molecular mass of the LMC was determined to be $\sim 30 \%$ of the neutral hydrogen mass and in the SMC only $\sim 7 \%$.

The NANTEN CO emission survey of the LMC confirmed the distribution of molecular gas as determined by Cohen et al. (1988). They disentagle the $\mathrm{CO}$ complexes in more than 100 molecular clouds with sizes ranging from 30 to 


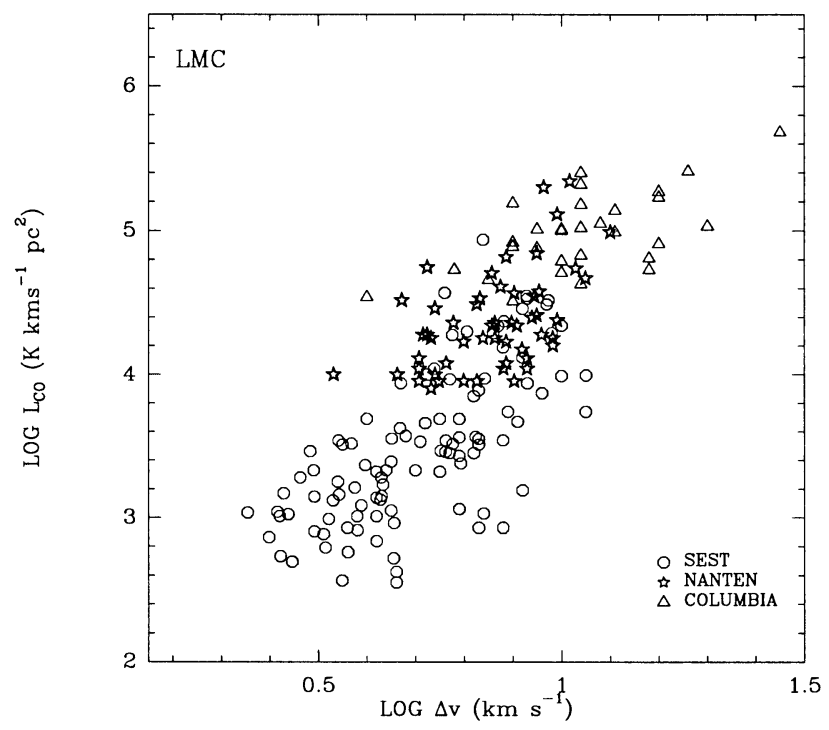

Figure 1. CO luminosity versus line width for molecular clouds in the LMC as defined by SEST(circles), NANTEN (stars) and Columbia (triangles) observations.

$100 \mathrm{pc}$ and virial masses exceeding $10^{4} \mathrm{M}_{\odot}$ (Fukui et al. 1999 and references therein). Assuming a conversion factor of 2 to 4 times larger than the Galactic factor they obtain a total molecular hydrogen mass of $7 \times 10^{7} \mathrm{M}_{\odot}$ which is about $10 \%$ of the total HI mass.

The SEST observations have resolved the Columbia molecular complexes into clouds of sizes of 10 to $40 \mathrm{pc}$ and virial masses of $(0.3$ to 5.5$) \times 10^{5} \mathrm{M}_{\odot}$. Determination of the physical properties of these molecular clouds is underway. In this regard, multiple-line observations in different transitions of the $\mathrm{CO}$ molecule as well as other molecules have been performed (e.g. Chin et al. 1998; Johansson et al. 1998).

Figure 1 shows the $L_{C O}$ versus $\Delta v$ realtionship for all the molecular clouds in the LMC as defined by the three sets of observations with different angular resolutions. These include about 100 molecular clouds from the SEST data (Rubio 1997; Kutner et al. 1998) and 55 clouds from the NANTEN survey. The SEST observtions appear concentrated in the lower-left part of the diagram while the Columbia observations are concentrated in the upper-right part. The NANTEN observations cover the range, both in $L_{C O}$ and $\Delta v$ in between the SEST (Johansson et al. 1998; Kutner et al. 1997; Rubio 1997) Columbia (Cohen et al. 1988).

Figure 2 shows the ratio $X / X_{g a l}$ as a funtion of cloud size, R, for SEST and NANTEN molecular clouds in the LMC, molecular clouds in the outer galaxy (May et al. 1997), and molecular clouds in the inner Galaxy (Bronfman et al., private communication), where $X_{\text {gal }}=2.3 \times 10^{20} \mathrm{~cm}^{-2}\left(K \mathrm{~km} \mathrm{~s}^{-1}\right)^{-1}$. The $X$ factor is defined as $X \equiv M\left(H_{2}\right) / L_{C O}$. To compute the $X$ factor we used 


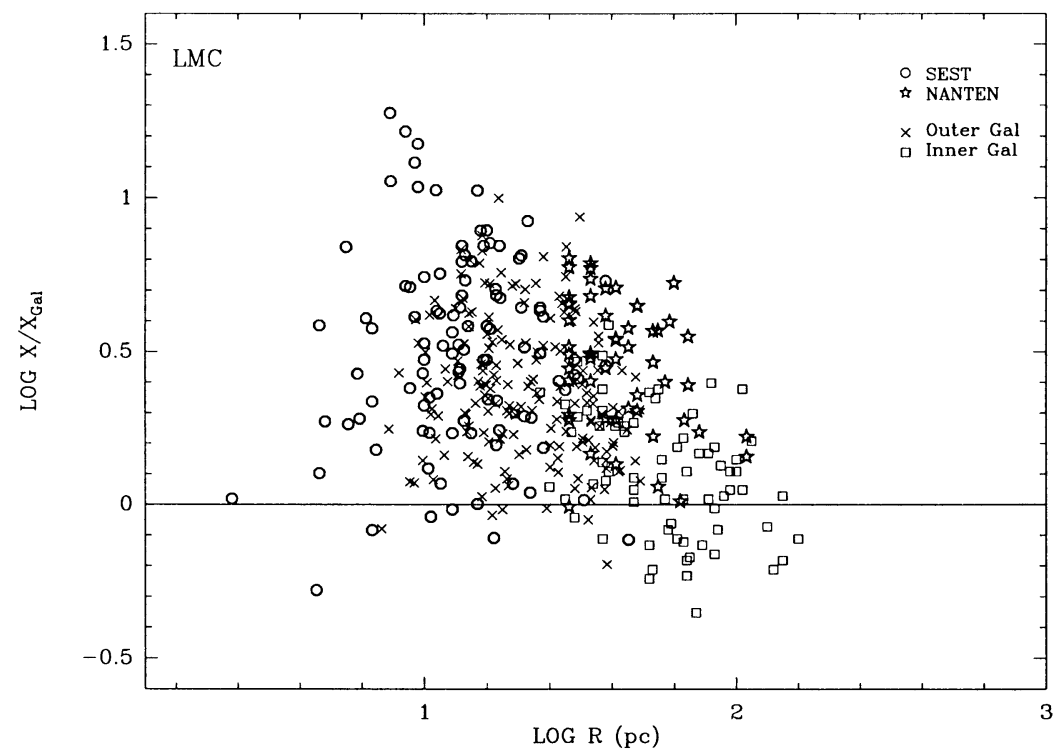

Figure 2. Ratio of the conversion factor $X / X_{\text {gal }}$ for molecular clouds in the LMC as defined by SEST(circles) and NANTEN (stars), outer Galaxy (crosses), and inner Galaxy (rectangles) versus size.

as mass the virial mass, $M_{v i r}=190 R \Delta v^{2}$. Although the points in this figure show a large spread, some trends are evident. Inner galaxy clouds tend to concentrate around the standard value for the Galaxy, $X_{\text {gal }}$, as determine by other methods (Strong et al. 1988), that is $X / X_{\text {gal }} \approx 1$. Outer galaxy clouds exhibit a larger spread, but with an average value of $\log \left[X / X_{\text {gal }}\right]=0.4$, indicating that the conversion factor is about two times larger than the Galactic value (May et al. 1997).

The CO clouds in the LMC have similar sizes as those of outer galaxy clouds. The difference between the size of SEST and NANTEN clouds is due solely to the different angular resolution of the observations. The SEST and NANTEN observations show a clear difference in the $X / X_{\text {gal }}$ value. Fukui et al. 1998 reports $X=(9 \pm 6) \times 10^{20} \mathrm{~cm}^{-2}\left(K_{\mathrm{km} \mathrm{s}}^{-1}\right)^{-1}$ about 2 to 4 times $X_{\text {gal }}$. SEST data indicates an average value of about 4 times larger than the standard Galactic value (Rubio 1997) although the spread in $X$ values is about one order of magnitude. An explanation of this large spread requires a detailed study of the local ambient conditions of the molecular clouds over the different regions in the LMC.

Comparison of the $\mathrm{CO}$ emission within one Columbia beam to that observed by SEST within the same region, shows that the CO luminosity measured in one Columbia beam is equal to the sum of the luminosities of the smaller clouds observed by SEST in the corresponding area (Kutner at al. 1997). This result confirms the suggestion that the molecular gas in the LMC is dominated by clouds, with no diffuse $\mathrm{CO}$ emission in the intercloud medium. Further work comparing the properties of the $\mathrm{CO}$ clouds as derived from these three sets 


\section{Doradus}

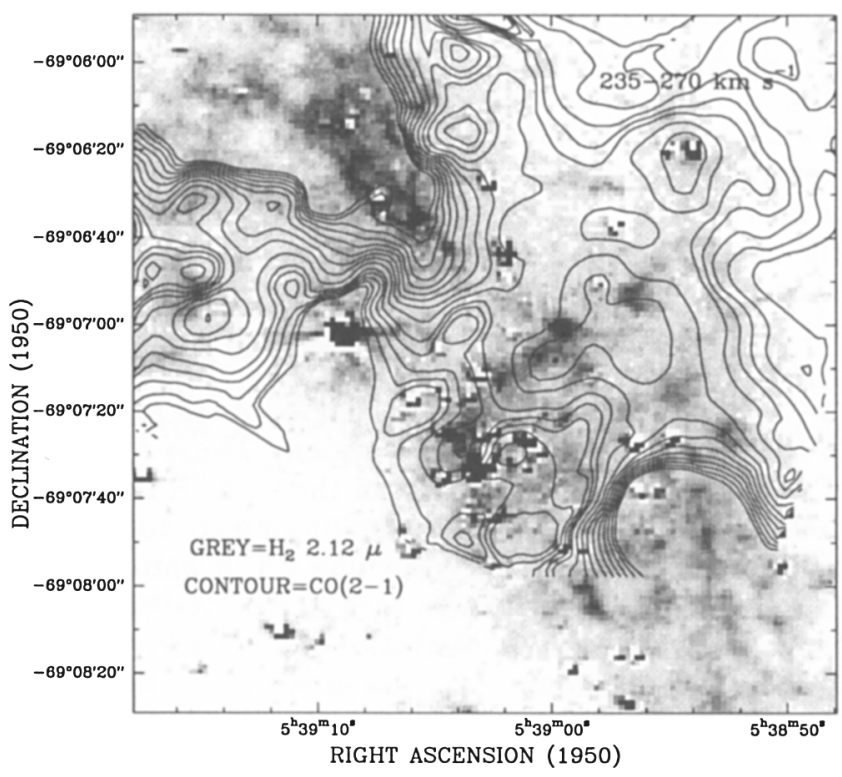

Figure 3. $2.12 \mu$ image of the 30 Doradus Nebula. Superimposed is the velocity integated $\mathrm{CO}(2-1)$ contour map.

of observations is envisaged as this comparison will be most valuable for the interpretation of extragalactic $\mathrm{CO}$ observations. This comparison should reveal the effects introduced in the determination of the properties of the molecular clouds when different spatial resolutions are used.

Our present knowledge of the molecular gas in the LMC indicates that the gas is dominated by clouds which are underluminous in $\mathrm{CO}$ and clumpy. The $\mathrm{CO}$ cloud properties are different from those of their galactic counterparts and this result is independent of the angular resolution used for the identification of the $\mathrm{CO}$ emission structures. The relation between the intensity of the $\mathrm{CO}$ emission and the molecular hydrogen density, the conversion factor $X$, is smaller than the factor derived for molecular clouds in our Galaxy. The $X$ value is dependent on the local ambient conditions of the $\mathrm{CO}$ clouds. Clearly, more work is needed to determine how local ambient conditions, e.g., photodissociation, metallicity, etc, affect the properties of the $\mathrm{CO}$ clouds.

\section{Near IR studies of the Molecular Gas in 30 Doradus}

An important result of the studies of the molecular gas in the Magellanic Clouds is that $\mathrm{CO}$ molecules can only survive in the dense cores of the interstellar medium while they are photodissociated in the less dense medium. (Rubio et al. 1993; Lequeux et al. 1995). Therefore, the interclump medium may contain large amounts of $\mathrm{H}_{2}$ as this molecule is more selfshielded against the UV field. 
Near IR studies of star forming regions in the LMC has been made through deep imaging in the $2.12 \mu \mathrm{H}_{2}$ 1-0 S(1) line and the $2.16 \mu \mathrm{Br} \gamma$ emission line by Probst \& Rubio (1997). The results obtained toward the giant HII region 30 Doradus are striking. The IR images show an intriguing molecular gas distribution. Figure 3 shows a $2.12 \mu$ image taken with CIRIM at the $1.5 \mathrm{~m}$ telescope at Cerro Tololo of the central $4.5 \times 4.5$ area of 30 Doradus. A chain of dense $\mathrm{H}_{2}$ knots are seen to the north of the central cluster R136 and a reticulated structure is seen towards the northeast and southwest regions of the nebula. $\mathrm{CO}(1-0)$ emission had been reported by Johansson et al. (1998) from two molecular clouds to the northeast and southwest of the big arcs and filaments delineated by the ionized hydrogen gas of the nebula. The detection of the molecular hydrogen knots where no $\mathrm{CO}$ emission had been reported led us to perform deep $\mathrm{CO}(2-1)$ observations in the central area of the HII region. These observations revealed the presence of weak, clumpy CO emission in the area (Rubio, Garay, \& Probst 1998). The velocity integrated $\mathrm{CO}(2-1)$ emission line contours have been superimposed on the $2.12 \mu$ image in Figure 3. The $2.12 \mu$ knots appear to be well correlated to the different $\mathrm{CO}(2-1)$ peaks seen in the contour map. This association is confirmed when the $\mathrm{CO}(2-1)$ emission is integrated in different velocity ranges. The $\mathrm{H}_{2}$ knots and condensation are seen on the border or the peak of the $\mathrm{CO}(2-1)$ emission.

The detection of compact $\mathrm{H}_{2}$ knots projected close to $\mathrm{R} 136$ seems to indicate that dense molecular stuctures can survive the strong winds and intense ionizing radiation produced by the luminous young stars of the compact R136 cluster.

Acknowledgments. We are grateful to the LOC and the IAU for their support to attend the meeting. This work has been funded through FONDECYT (CHILE) grant $\mathrm{N}^{\circ} 1960926$.

\section{References}

Combes, F. 1991, ARA\&A, 29, 41

Cohen, R.S., Dame, T.M., Garay, G., at al. 1988, ApJ, 331, L95

Chin, Y. 1999, this volume

Fukui, Y., Abe, R., Hara, A., et al. 1999, this volume

Israel, F.P., Johansson, L.E.B., Lequeux, J., et al. 1993, A\&A, 276, 25

Johansson, L.E.B., Greve, A., Booth, R.S., et al. 1998, A\&A, 331, 857

Johansson, L.E.B., Heikkila, A., \& Olofsson, H. 1999, this volume

Kutner, M.L., Rubio, M., Booth R.S., et al. 1997, A\&AS, 122, 255

Kutner, M.L., Rubio, M., Chair, J., et al. 1999, In preparation

Lequeux, J., Le Bourlot, J., Pineau des Forets, G., et al. 1994, A\&A, 292, 371

May, J, Alvarez, H., \& Bronfman, L. 1997, A\&A, 327, 325

Probst R., \& Rubio, M. 1997, BAAS, vol 29, 834

Rubio, M. Garay, G., Montani, J., et al. 1991, ApJ, 368, 173

Rubio, M. Lequeux, J., \& Boulanger, F. 1993, A\&A, 271, 9

Rubio M., Garay, G., \& Probst R. 1998, The Messenger, vol 93, p.34 
Rubio, M. 1997, in IAU Symposium 170: CO:Twenty-five years of Millimeterwave Spectroscopy, eds. W.B. Latter et al., Kluwer, p. 265.

Strong, A.W., Bloemen J.B.G.M., Dame, T.M., et al. 1988, A\&A, 207, 1

Young, J.S., \& Scolville, N. 1991, ARA\&A, 29, 581

\section{Discussion}

Jan Palous: Is it possible, or how far it is possible, to use the virial masses of $\mathrm{CO} / \mathrm{H}_{2}$ clouds in regions of star formation and new clusters? The clouds are probably expanding and out of equilibrium. Is it not possible that a fraction of the difference in $\mathrm{X}$ factor is due to more non-equilibrium clusters in the LMC and the SMC than in the MW?

Rubio: In our galaxy, the virial mass determination gives similar results as the masses derived from the $\mathrm{CO}$ luminosity applying the galactic $\mathrm{X}$ factor. This result includes molecular clouds in the star forming regions, which may not be in virial equilibrium. The interesting result is that when we apply the galactic $\mathrm{X}$ factor to the $\mathrm{CO}$ luminosities measured in the Magellanic Clouds the derived masses are lower than those determined from the virial mass.

It would be an interesting exercise to see if there is a significant difference between the value of the $\mathrm{X}$ factor for molecular clouds associated with star forming regions in the galaxy and those that are not associated with star formation.

Since we cannot measure the $\mathrm{H}_{2}$ directly we have to look for alternative ways to determine the total $\mathrm{H}_{2}$ mass. Using the virial mass, at least, in our galaxy gives consistent results to other methods used.

Margarita Rosado: Monica, could you tell us which is the $\mathrm{X}=\mathrm{H}_{2} / \mathrm{CO}$ fraction derived directly from your $2.12 \mu\left(\mathrm{H}_{2}\right)$ and SEST $(\mathrm{CO})$ observations in 30 Dor, and how does it compare with the previous estimates of $\mathrm{X}$ ?

Rubio: The $\mathrm{X}$ factor derived from the $\mathrm{CO}$ luminosity and the virial determination of the mass of these knots is larger than the $\mathrm{X}$ factor derived for the ensemble of $\mathrm{CO}$ clouds mapped by SEST. It can be as high as 20 times the galactic conversion factor. To derive a mass from the $2.12 \mu$ flux we need to know the excitation mechanism for the $\mathrm{H}_{2}$. We will have a spectroscopic run next semester to investigate this mechanism. Anyhow, the column density that we can derive from the $2.12 \mu \mathrm{H}_{2}$ emission will be of the hot gas and not the cold $\mathrm{H}_{2}$ gas determined by the $\mathrm{CO}$ molecule. It will be an interesting result to compare with PDR's models that can give the fraction of warm to cold molecular gas.

Klaas de Boer: Since the "X-Factor" has been mentioned several times. I would like to comment on it. The $\mathrm{X}$ is defined as $\mathrm{N}\left(\mathrm{H}_{2}\right) / \mathrm{I}(\mathrm{CO})$. However, the $\mathrm{H}_{2}$ is not a measured parameter, it merely is derived from the far-infared luminosity by assuming a constant factor $\mathrm{L}(\mathrm{IR})$ to $\mathrm{N}\left(\mathrm{H}_{2}\right)$. So all the discussion about variation in $\mathrm{X}$ has nothing to do with $\mathrm{H}_{2}$, but only with variation of $\mathrm{L}(\mathrm{CO})$. I would like to see the use of this $\mathrm{X}$-factor be ended, but for historical reasons this will probably not happen. 